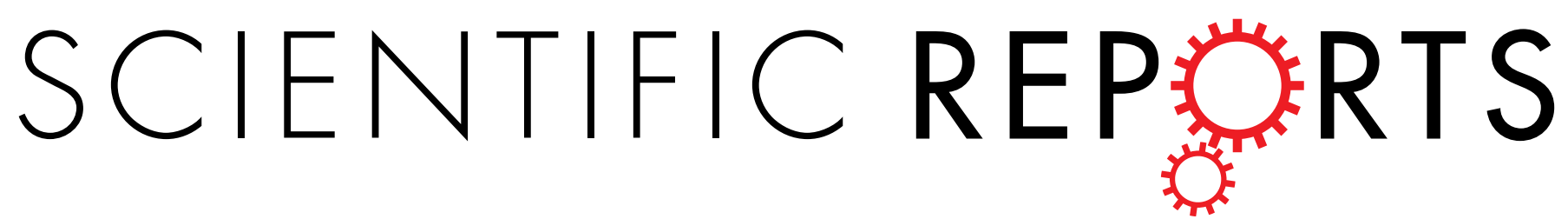

Received: 13 February 2019

Accepted: 3 June 2019

Published online: 20 June 2019

\section{Frequency of the CYP 2 C $19 * 17$ polymorphism in a Chilean population and its effect on voriconazole plasma concentration in immunocompromised children}

\author{
N. Espinoza ${ }^{1}$, J. Galdames ${ }^{1}$, D. Navea ${ }^{1}$, M. J. Farfán ${ }^{1,2} \&$ C. Salas ${ }^{1}$ \\ Invasive fungal infections (IFIs) are the most frequent cause of morbidity and mortality in \\ immunocompromised children. Voriconazole is the first-line antifungal choice in the treatment of \\ IFIs like aspergillosis. Voriconazole pharmacokinetics vary widely among patients and voriconazole is \\ metabolized mainly in the liver by the CYP2C19 enzyme, which is highly polymorphic. The CYP2C19*17 \\ allele is characterized by the presence of four single nucleotide polymorphisms expressing an ultra- \\ rapid enzyme phenotype with an accelerated voriconazole metabolism, is associated with low \\ (sub-therapeutic) plasma levels in patients treated with the standard dose. Considering that in our \\ center a high percentage of children have sub-therapeutic levels of voriconazole when treated with \\ standard doses, we sought to determine the frequency of the CYP2C19*17 polymorphism (rs12248560) \\ in a Chilean population and determine the association between voriconazole concentrations and \\ the rs 12248560 variant in immunocompromised children. First, we evaluated the frequency of the \\ rs12248560 variant in a group of 232 healthy Chilean children, and we found that 180 children (77.6\%) \\ were non-carriers of the rs 12248560 variant, 49 children (21.1\%) were heterozygous carriers for \\ rs12248560 variant and only 3 children (1.3\%) were homozygous carriers for rs 12248560 variant, \\ obtaining an allelic frequency of $12 \%$ for variant in a Chilean population. To determine the association \\ between voriconazole concentrations and the rs 12248560 variant, we analyzed voriconazole plasma \\ concentrations in a second group of 33 children treated with voriconazole. In these patients, carriers \\ of the rs12248560 variant presented significantly lower voriconazole plasma concentrations than \\ non-carriers $(p=0,011)$. In this study, we show the presence of the rs 12248560 variant in a Chilean \\ population and its accelerating effect on the pharmacokinetics of voriconazole in pediatric patients. \\ From these data, it would be advisable to consider the variant of the patient prior to calculating the \\ dosage of voriconazole.
}

Pediatric patients diagnosed with hemato-oncological diseases or who have had a solid organ transplant must undergo prolonged periods of chemotherapy and immunosuppressive therapy, which makes these patients susceptible to invasive fungal infections (IFIs) ${ }^{1,2}$. These infections are caused by the colonization of pathogens of the genus Aspergillus and Candida and are an important cause of morbimortality in pediatric patients. Overcoming these infections is crucial to the survival of these patients $\mathrm{s}^{3,4}$.

Voriconazole is a broad-spectrum anti-fungal drug that belongs to the family of second-generation triazoles. It is used as a first-line treatment for IFIs, characterized by a high inter- and intra-patient pharmacokinetic variability. This condition makes its dosage difficult, which is why permanent therapeutic drug monitoring (TDM) is needed, focused mainly on achieving the correct dosage to obtain therapeutic plasma concentrations ${ }^{5,6}$.

${ }^{1}$ Laboratorio Clínico, Hospital Dr. Luis Calvo Mackenna, Santiago, Chile. ${ }^{2}$ Departamento de Pediatría y Cirugía Infantil, Campus Oriente, Hospital Dr. Luis Calvo Mackenna, Facultad de Medicina, Universidad de Chile, Santiago, Chile. Correspondence and requests for materials should be addressed to M.J.F. (email: mfarfan@med.uchile.cl) or C.S. (email: csalas@calvomackenna.cl) 


Group 1:
Healthy children
$\mathrm{n}=232$$\rightarrow \begin{gathered}\text { Inclusion/exclusion } \\ \text { criteria and } \\ \text { sign consent/asent }\end{gathered} \rightarrow \begin{aligned} & \text { Blood } \\ & \text { sample }\end{aligned} \rightarrow$ PCR/RFLP $\rightarrow \rightarrow \begin{gathered}\text { Allele frequency of } \\ \text { rs12248560 } \\ \text { variant }\end{gathered}$

\begin{tabular}{|c|c|c|c|c|c|c|c|}
\hline $\begin{array}{l}\text { Group 2: } \\
\text { Pediatric patients }\end{array}$ & & Inclusion/exclusion & $\begin{array}{l}\text { Blood } \\
\text { sample }\end{array}$ & $\rightarrow$ & HPLC & $\rightarrow$ & $\begin{array}{l}\text { Voriconazole trough } \\
\text { plasma concentrations }\end{array}$ \\
\hline $\begin{array}{l}\text { with voriconazole } \\
\qquad \mathrm{n}=33\end{array}$ & & sign consent/asent & $\begin{array}{c}\text { Blood } \\
\text { sample or } \\
\text { nasopharyngeal } \\
\text { swabs }\end{array}$ & $\rightarrow$ & PCR/RFLP & $\rightarrow$ & $\begin{array}{c}\text { Detection of } \\
\text { rs12248560 } \\
\text { variant }\end{array}$ \\
\hline
\end{tabular}

Figure 1. Methodological scheme of the study design.

Voriconazole is metabolized in the liver, mainly by two cytochrome P450 enzymes (CYP), CYP2C19 and CYP3A4, and flavin-containing monooxygenase 3 (FMO3) ${ }^{7,8}$ converting it to N-oxide voriconazole. This compound has minimal anti-fungal activity. The CYP2C19 gene is highly polymorphic; to date more than 30 single nucleotide polymorphisms (SNPs) have been identified in this gene. However, few of these have been associated with any clinical response. The CYP2C19*17 allele is characterized by the presence of four SNPs $(-3402 \mathrm{C}>\mathrm{T}$, $-806 \mathrm{C}>\mathrm{T}, 99 \mathrm{C}>\mathrm{T}, 80161 \mathrm{~A}>\mathrm{G}(\mathrm{I} 331 \mathrm{~V}))$. Also, CYP2C19*17 allele is also present in a low function allele $C Y P 2 C 19 * 4 B^{9}$. Several studies have shown that the presence of $-806 \mathrm{C}>\mathrm{T}$ SNP (rs12248560) is correlated with lower plasma concentrations of voriconazole ${ }^{6,9-13}$. The rs 12248560 variant is associated with an ultra-rapid metabolizer phenotype of enzyme CYP2C19, which translates into a faster elimination of the drug in patients who receive treatment with standard doses of voriconazole with the consequent risk of therapeutic failure, making dosage corrections necessary ${ }^{14-16}$. Little is known of the effect of rs 12248560 variant on pediatric patients; it is possible that genetic variants present in patient carriers could have a significant clinical impact on treatment with voriconazole, resulting in the spread of the infection and deterioration of the patient.

In Chile, there is no information about the frequency of rs12248560 variant and its impact on voriconazole pharmacokinetics. The aim of this work was to describe the frequency of rs 12248560 variant in a Chilean population and to study its association with the pharmacokinetics of voriconazole in pediatric patients.

\section{Material and Methods}

Patients. In this work we analyzed two groups of children (Fig. 1). In order to determine the frequency of the rs 12248560 variant in a Chilean population, a group of healthy children was enrolled (group 1). To evaluate the effect of the rs12248560 variant on voriconazole plasma concentrations, we included a group of immunocompromised children treated with voriconazole (group 2).

For group 1, 232 samples were collected from healthy Chilean children who attend the Dr. Luis Calvo Mackenna hospital (HLCM) for routine clinical testing. Children being treated with voriconazole were excluded. The ABO blood phenotype was obtained in 145 (63\%) children in this group.

For group 2, 33 patients were included who received treatment with voriconazole, administered intravenously (IV) or orally (PO) between 2015 and 2017 years at the HLCM, Roberto del Río (HRR) and Base de Valdivia (HBV) hospitals. We excluded patients who presented pre-existing hepatic toxicity to treatment with voriconazole.

All patients and/or parents from groups 1 and 2 provided written informed consent to participate in the study approved by the Pediatric Scientific Ethics Committee of the Metropolitan East Health Service, the Ethics Committee of the Metropolitan North Health Service and the Valdivia Health Service. In addition, all methods were performed in accordance with Declaration of Helsinki.

Nucleic acid extraction. Nucleic acids were extracted from blood samples using QIAamp ${ }^{\circledR}$ DNA blood Mini Kit (Qiagen) following the manufacturer's instructions. For bone marrow transplant patients, the nucleic acids were extracted from nasopharyngeal swabs using the MagNa Pure Kit (Roche) according to the manufacturer's instructions. Purified nucleic acids were stored at $-20^{\circ} \mathrm{C}$ for later use.

Polymorphisms detection. PCR-RFLP was used to detect rs 12248560 variant as described ${ }^{3}$. For the PCR amplification, the GoTaq ${ }^{\circledR}$ Flexi DNA polymerase enzyme from Promega was used. The restriction enzyme LweI was purchased from Thermo Fisher Scientific and used following the supplier's specifications. The digestion fragments were visualized in $10 \%$ polyacrylamide gels. PCR-RFLP methods were validated by sequencing.

Determination of the voriconazole plasma concentrations. For TDM, blood samples were taken in steady-state one hour before voriconazole administration according to the clinical protocols for antifungal treatment in each hospital. Voriconazole plasma concentration was determined by high performance liquid chromatography (HPLC) according to a previously validated method ${ }^{17}$ using an Agilent 1260 Infinity HPLC system with a VL diode array detector. Ketoconazole was used as the internal standard. Each run was done at $35^{\circ} \mathrm{C}$ in the column with isocratic elution, a flow of $1 \mathrm{~mL} / \mathrm{min}, 20 \mathrm{uL}$ of sample injection and measured at a wavelength of 254 nanometers. Elution time for voriconazole and ketoconazole were 3.3 and 4.4 minutes, respectively. Recovery percentage of voriconazole was $101.6 \% \pm 2 \%$ and both the repeatability $(0.97 \%)$ and the reproducibility $(1.73 \%$ and $1.77 \%$ ) of the method were less than $2 \%$. 


\begin{tabular}{|c|c|c|c|}
\hline & Non-Carriers & Carriers & $p$ Value \\
\hline Total patients & 22 & 11 & \\
\hline Female & 10 & 7 & \multirow{2}{*}{0.465} \\
\hline Male & 12 & 4 & \\
\hline Age median (years) [IQR] & $7.5[2.8-10.5]$ & $9[7,8-10,5]$ & 0.135 \\
\hline Weight median $(\mathrm{kg})[\mathrm{IQR}]$ & $23.5[14,1-28,8]$ & $27.7[25,0-32,0]$ & 0.0217 \\
\hline Plasma concentrations measurement/patients median [IQR] & $1.5[1-2,75](n=22)$ & $1.0[1-2](n=11)$ & 0.615 \\
\hline Dose median (mg/kg/day) [IQR] & $14.9[10,0-16,0](n=81)$ & $14.6[9,3-18,0](n=49)$ & 0.9 \\
\hline All the dose-corrected plasma concentrations $(\mu \mathrm{g} / \mathrm{mL} / \mathrm{mg} / \mathrm{kg} /$ day) mean [SD] & $0.046[0,174](n=81)$ & $0.034[0,07](n=49)$ & 0.011 \\
\hline First voriconazole plasma concentration median $(\mu \mathrm{g} / \mathrm{mL})[\mathrm{IQR}]$ & $1.12[0,39-3,92](n=22)$ & $0.37[0,16-1,19](n=11)$ & 0.054 \\
\hline First dose-corrected voriconazole concentration median $(\mu \mathrm{g} / \mathrm{mL} / \mathrm{mg} / \mathrm{kg} / \mathrm{day})[\mathrm{IQR}]$ & $0.10[0,02-0,34](n=22)$ & $0.029[0,02-0,08](n=11)$ & 0.059 \\
\hline \multicolumn{4}{|l|}{ Diagnosis } \\
\hline ALL & 13 & 6 & \\
\hline AML & 5 & 5 & \\
\hline Osteosarcoma & 1 & - & \\
\hline Liver transplant & 1 & - & \\
\hline Testicle tumor & 1 & - & \\
\hline SCID & 1 & - & \\
\hline
\end{tabular}

Table 1. Demographic and pharmacokinetic data of patients with voriconazole treatment. IQR, interquartile range; SD, Standard deviation; ALL, acute lymphocytic leukemia; AML, acute myeloid leukemia; SCID, Severe Combined Immunodeficiency.

Statistical analysis. To determine the allelic frequency of the rs12248560 variant in a Chilean population (group 1), a sample size of 232 samples was calculated, assuming a frequency of this variant of $20 \%$ described for Caucasian population $^{18}$, with $80 \%$ power, $5 \%$ error and a $95 \%$ confidence level. To compare the gender distribution between carriers and non-carriers of group 2, we used Fisher's test. For the gender and ABO blood group comparison, as well as determination of the Hardy-Weinberg equilibrium, we used the chi-squared statistical test $\left(X^{2}\right)$. To determine the influence of the rs 12248560 variant on voriconazole plasma concentrations, since one subject can have multiple plasma levels, a multilevel generalized linear model was created for correlated samples with gamma distribution and log link identity (distribution of the plasma concentration adjusted for dose and weight). A significant $p$ value $<5 \%$ was considered. To determine the influence of the rs 12248560 variant on the first voriconazole plasma level and to compare plasma concentrations measurements per patients, the Mann-Whitney statistical test was used. Statistical analysis was performed with Stata v.12.1 (StataCorp, 2011. Stata Statistical Software: Release 12. College Station, TX: StataCorp LP.).

\section{Results}

Allelic frequency of the rs12248560 variant in a Chilean population. Of the 232 children analyzed in group $1,49.5 \%$ (115 patients) were girls, with an average of $5.5 \pm 5.2$ years of age. We found that 180 children (77.6\%) were non-carriers of the rs12248560 variant, 49 children (21.1\%) were heterozygous carriers for rs 12248560 variant and only 3 children (1.3\%) were homozygous carriers for rs 12248560 variant (Table 1). With these genotypic frequencies, an allelic frequency of $12 \%$ for the rs 12248560 variant was obtained. In addition, it was established that this population is in the Hardy-Weinberg equilibrium.

No statistical differences were found in the ABO blood group between the children included in this study and a Chilean population, indicating that the study population was representative (Supplementary Fig. 1).

Allelic frequency of the rs12248560 variant in patients treated with voriconazole. The presence of the rs12248560 variant was studied in 33 patients treated with voriconazole (group 2). The demographic and pharmacokinetic data are shown in Table 1 . The rs 12248560 variant was found that $66.7 \%$ (22 patients) of the patients were non-carriers of rs 12248560 variant, $27.3 \%$ (9 patients) were heterozygous carriers of rs 12248560 variant and $6 \%$ (2 patients) were homozygous carriers of rs12248560 variant.

Carrier of rs12248560 variant presented lower voriconazole plasma concentrations than non-carriers. We compared 81 dose-corrected voriconazole plasma concentrations of 22 non-carriers of rs12248560 variant and 49 plasma concentrations from 11 carriers of the rs 12248560 variant. It is important to note, that patients included in our study were grouped by presence or absence of the rs 12248560 variant (carriers and non-carriers) and not based on CYP2C19 phenotype, since only two patients were homozygous for the rs12248560 variant with one level each. We found that carriers of the rs12248560 variant presented significantly lower voriconazole plasma concentrations than non-carriers ( $p=0,011$; Fig. $2 \mathrm{~A})$.

The voriconazole plasma concentrations without dose correction were studied (first plasma level). It was observed that almost all the carriers of the rs12248560 variant presented the first sub-therapeutic trough plasma concentration with a median of $0.37 \mu \mathrm{g} / \mathrm{mL}$, whereas in the non-carriers there was a wide spread in the concentrations with a median of $1.12 \mu \mathrm{g} / \mathrm{mL}$ (Fig. 2B). 

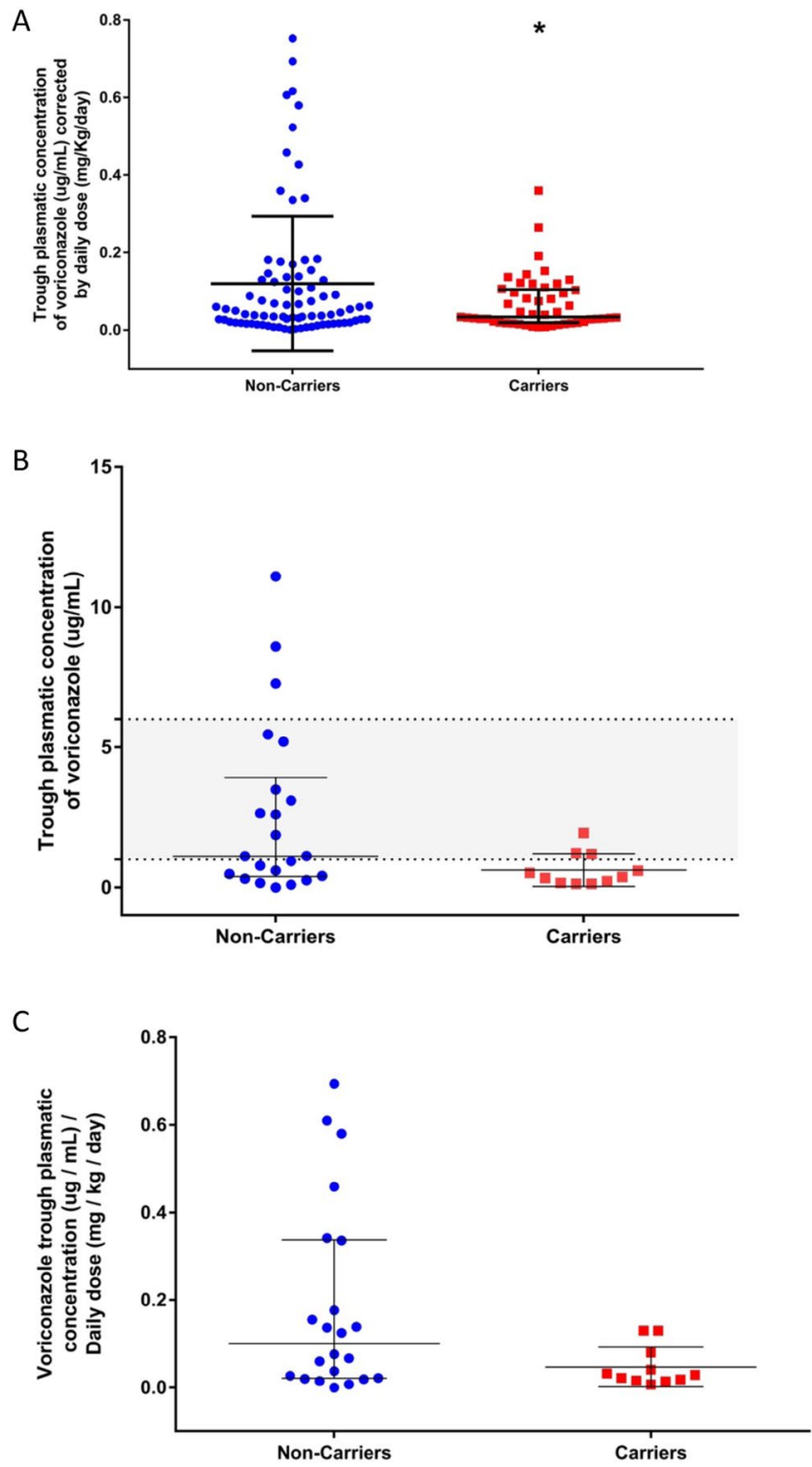

Figure 2. Voriconazole plasma concentration in carriers or non-carriers of the rs12248560 variant. (A) Dosecorrected voriconazole plasma concentrations of the 33 patients are shown. The carriers had a mean of $0.034 \mu \mathrm{g} /$ $\mathrm{mL} / \mathrm{mg} / \mathrm{kg} /$ day and showed significantly lower concentrations than non-carriers $(p=0,011)$, whose mean was $0.046 \mu \mathrm{g} / \mathrm{mL} / \mathrm{mg} / \mathrm{kg} /$ day. The mean and the standard deviation for each genotype are plotted. (B) First trough plasma concentrations of voriconazole, without dose correction, reached by the patients studied $(p=0.054)$. Almost all the concentrations belonging to the carriers of the rs12248560 variant were sub-therapeutic (median $0.37 \mu \mathrm{g} / \mathrm{mL}$ ), whereas for the non-carriers the concentrations were spread out (median $1.12 \mu \mathrm{g} / \mathrm{ml}$ ). The medians and interquartile ranges are plotted and the therapeutic range (between 1 and $6 \mu \mathrm{g} / \mathrm{mL}$ ) is highlighted. (C) First dose-corrected voriconazole concentration of the 33 patients is shown. The median for each genotype were 0.10 and $0.029 \mu \mathrm{g} / \mathrm{mL} / \mathrm{mg} / \mathrm{kg} /$ day for non-carriers and carriers of the rs 12248560 variant, respectively $(p=0.059)$.

Finally, the first trough voriconazole dose-corrected plasma concentrations for each genotype were compared. The carriers of the rs12248560 variant had a median $0.029 \mu \mathrm{g} / \mathrm{mL} / \mathrm{mg} / \mathrm{kg} /$ day, whereas the non-carriers had $0.10 \mu \mathrm{g} / \mathrm{mL} / \mathrm{mg} / \mathrm{kg} /$ day $(p=0.059 ;$ Fig. $2 \mathrm{C})$.

\section{Discussion}

In this study we examined the association of the rs12248560 variant on voriconazole plasma concentrations in immunocompromised Chilean pediatric patients. In addition, the allelic frequency of this variant in a Chilean population was obtained. The rs12248560 variant has been studied in other populations ${ }^{19}$. In a population of 285 subjects in the north of Spain, $14.9 \%$ of them were carriers of the rs 12248560 variant, a value similar to the $12 \%$ obtained in our study ${ }^{20}$. Similarly, data obtained from the 1000 Genomes Project showed a frequency $12 \%$ of the rs12248560 variant in the American population. In another study in the north (Denmark, Sweden, Norway), east (Poland) and south (France) of Europe, the allelic frequencies of this variant was between 15 and $27 \%$. This frequency was similar to what has been described in Africa (Ethiopian, Nigerian, Afro-American), a frequency 
reported as being between 18 and 20\%, whereas for Asian groups (Korean, Chinese and Japanese), frequencies between 2 and $4 \%$ were described ${ }^{21}$.

We found differences in voriconazole plasma concentrations in carriers of the rs12248560 variant compared to non-carriers. Also, analyzing the first trough voriconazole plasma concentrations revealed that plasma concentrations found in rs12248560 variant carriers were lower than the non-carriers. The same association has been reported in immunosuppressed hemato-oncology pediatric patients ${ }^{6,12}$, demonstrating that the heterozygote patients have lower plasma concentrations than non-carriers. In another study performed on 72 adult patients with aspergillosis ${ }^{22}$, it was determined that rs 12248560 variant carriers presented statistically lower voriconazole plasma concentrations than non-carriers. The pharmacokinetics of voriconazole in 20 healthy adults after a single oral dose of $200 \mathrm{mg}$ showed that rs 12248560 variant carriers had a maximum concentration, AUC and clearance of voriconazole significantly lower than non-carriers ${ }^{10}$.

Early determination of the allelic rs 12248560 variant and its consideration in guiding the voriconazole dosage in patients made it possible to reduce the time needed to reach therapeutic concentrations from 29 to 6.5 days in carriers of the rs 12248560 variant and from 25 to 9 days in homozygous patients for the wild-type variant CYP2C19*1. In addition, there was a reduction in patients with an elevation of liver enzymes and suspension of the treatment due to toxicity ${ }^{12}$. A recent report established that the CYP2C19 enzyme genotype is the most important interpersonal variability factor that affects the plasma concentrations of voriconazole ${ }^{23}$. In this regard, clinical guidelines for voriconazole therapy based on the CYP2C19 genotype have recently been published providing evidence for the use and dosage of this $\mathrm{drug}^{19}$. Implementation of genotype-targeted therapy together with adequate therapeutic monitoring in accordance with clinical recommendations could produce even shorter dwell times or reduce the hospital stay, avoid transfer to high complexity units and prolonged drug treatment with voriconazole, lower the request for imaging or laboratory examinations, and so forth.

Our study has limitations. An interindividual variability in voriconazole plasma levels has been described. Age, gender, drug route and some co-morbidities have been shown to affect the voriconazole plasma concentration ${ }^{19,24,25}$. In our study no differences were found in age and gender between carriers and non-carriers of the rs12248560 variant in patients treated with voriconazole (Table 1). Drug route and co-morbidities were not analyzed. Another limitation is that drug interactions, efficacy and toxicity of voriconazole and its association with clinical outcome were not analyzed in our study. Is important to note, in this study we did not analyze the involvement of others CYP2C19 variants or others enzymes (CYP3A4 and FMO3) that affects the plasma concentrations of voriconazole that might explain similar plasma concentrations between carriers and non-carriers of rs 12248560 variant. The main goal of our study was to find evidence of the impact of the rs 12248560 variant on the voriconazole plasma concentration. Future prospective age-matched studies including the variables mentioned above are needed to validate our findings in order to consider CYP2C19*17 genotyping for voriconazole therapeutic monitoring.

In conclusion, we report the frequency of the rs 12248560 variant in a Chilean pediatric population and the involvement of this variant in the voriconazole metabolism of immunocompromised patients. Our data might help in the implementation of CYP2C19 genotyping to individualize starting doses of voriconazole.

\section{References}

1. Zwitserloot, A. et al. Importance of neutropenia for development of invasive infections at various phases of treatment for hematooncological diseases in children. Scand J Infect Dis. 44(5), 355-362, https://doi.org/10.3109/00365548.2011.641505 (2012).

2. Döring, M. et al. Antifungal prophylaxis with posaconazole vs. fluconazole or itraconazole in pediatric patients with neutropenia. Eur J Clin microbiol 34(6), 1189-1200, https://doi.org/10.1007/s10096-015-2340-y (2015).

3. Baldwin, R. et al. Increased omeprazole metabolism in carriers of the CYP2C19*17 allele; a pharmacokinetic study in healthy volunteers. Br J Clin Pharmacol. 65(5), 767-774, https://doi.org/10.1111/j.1365-2125.2008.03104 (2008).

4. Omrani, A. S. \& Almaghrabi, R. S. Complications of hematopoietic stem transplantation: Fungal infections. Hematol Oncol Stem Cell Ther. 10(4), 239-244, https://doi.org/10.1016/j.hemonc.2017.05.013 (2017).

5. Tucker, L. et al. Voriconazole monitoring in children with invasive fungal infections. J Pediatr Pharmacol Ther. 20(1), 17-23, https:// doi.org/10.5863/1551-6776-20.1.17 (2015).

6. Hicks, J. et al. Voriconazole plasma concentrations in immunocompromised pediatric patients vary by CYP2C19 diplotypes. Pharmacogenomics J. 15(8), 1065-78, https://doi.org/10.2217/pgs.14.53 (2014).

7. Yanni, S. B. et al. In Vitro metabolism explains higher clearance of voriconazole in children versus adult: role of CYP2C19 and Flavin-containing monooxygenase 3. Drug Metab and Dispos. 38(1), 25-31, https://doi.org/10.1124/dmd.109.029769 (2010).

8. Zane, N. R. \& Thakker, D. R. A physiologically based pharmacokinetic model for voriconazole disposition predicts intestinal firstpass metabolism in children. Clin Pharmacokinet. 53(12), 1171-82, https://doi.org/10.1007/s40262-014-0181-y (2014).

9. Scott, S. A. et al. Identification of CYP2C19*4B: pharmacogenetic implications for drug metabolism including clopidogrel responsiveness. Pharmacogenomics J. 12(4), 297-305, https://doi.org/10.1038/tpj.2011.5 (2012).

10. Wang, G. et al. The CYP2C19 ultra-rapid metabolizer genotype influences the pharmacokinetics of voriconazole in healthy male volunteers. Eur J Clin Pharmacol. 65(3), 281-285, https://doi.org/10.1007/s00228-008-0574-7 (2009).

11. Dolton, M. \& McLachlan, A. Clinical importance of the CYP2C1917 variant allele for voriconazole. Br J Clin Pharmacol. 71(1), 137-138, https://doi.org/10.1111/j.1365-2125.2010.03801 (2011).

12. Teusink, A. et al. Genotype-Directed Dosing Leads to Optimized Voriconazole Levels in Pediatric Patients Receiving Hematopoietic Stem Cell Transplantation. Biol Blood Marrow Transplant. 22(3), 482-6, https://doi.org/10.1097/INF.0000000000001339 (2016)

13. Hamadeh, I. S. et al. Impact of the CYP2C19 genotype on voriconazole exposure in adults with invasive fungal infections. Pharmacogenet Genomics. 27(5), 190-196, https://doi.org/10.1097/FPC.0000000000000277 (2017).

14. Chuwongwattana, S. et al. A prospective observational study of CYP2C19 polymorphisms and voriconazole plasma level in adult Thai patients with invasive aspergillosis. Drug Metab Pharmacokinet. 31(2), 117-22, https://doi.org/10.1016/j.dmpk.2015.12.005 (2016).

15. Hirota, T., Eguchi, S. \& Ieiri, I. Impact of genetic polymorphisms in CYP2C9 and CYP2C19 on the pharmacokinetics of clinically used drugs. Drug Metab Pharmacokinet. 28(1), 28-37 (2013).

16. Mangal, N. et al. Optimization of Voriconazole Therapy for the Treatment of Invasive Fungal Infections in Adults. Clin Pharmacol Ther. 104(5), 957-965, https://doi.org/10.1002/cpt.1012 (2018).

17. Pennick, G., Clark, M., Sutton, D. \& Rinaldi, M. Development and validation of a high-performance liquid chromatography assay for voriconazole. Antimicrob Agents Chemother. 47(7), 2348-50, https://doi.org/10.1128/AAC.47.7.2348-2350.2003 (2003). 
18. Myrand, S. P. et al. Pharmacokinetics/genotype associations for major cytochrome P450 enzymes in native and first- and thirdgeneration Japanese populations: comparison with Korean, Chinese, and Caucasian populations. Clin Pharmacol Ther. 84(3), 347-61, https://doi.org/10.1038/sj.clpt.6100482 (2008).

19. Moriyama, B. et al. Clinical Pharmacogenetics Implementation Consortium (CPIC) Guidelines for CYP2C19 and Voriconazole Therapy. Clin Pharmacol Ther. 102(1), 45-51, https://doi.org/10.1002/cpt.583 (2016).

20. Vicente, J. et al. Genetic polymorphisms of CYP2C8, CYP2C9 and CYP2C19 in Ecuadorian Mestizo and Spaniard populations: A comparative study. Mol Biol Rep. 41(3), 1267-1272, https://doi.org/10.1007/s11033-013-2971-y (2014).

21. Lee, S. Clinical application of CYP2C19 pharmacogenetics toward more personalized medicine. Front Genet. 3(318), 1-7, https:// doi.org/10.3389/fgene.2012.00318 (2013).

22. Chawla, P. et al. Correlation of CYP2C19 genotype with plasma voriconazole levels: a preliminary retrospective study in Indians. Int J Clin Pharm. 37(5), 925-930, https://doi.org/10.1007/s11096-015-0143-y (2015)

23. Zhong, X., Tong, X., Ju, Y., Du, X. \& Li, Y. Interpersonal Factors in the Pharmacokinetics and Pharmacodynamics of Voriconazole: Are CYP2C19 Genotypes Enough for Us to Make a Clinical Decision? Curr Drug Metab. 19(14), 1152-1158, https://doi.org/10.217 4/1389200219666171227200547 (2018).

24. Allegra, S. et al. Pharmacogenetic of voriconazole antifungal agent in pediatric patients. Pharmacogenomics J. 19(11), 913-925, https://doi.org/10.2217/pgs-2017-0173 (2018).

25. Allegra, S. et al. Therapeutic drug monitoring of voriconazole for treatment and prophylaxis of invasive fungal infection in children. Br J Clin Pharmacol. 84(1), 197-203, https://doi.org/10.1111/bcp.13401 (2018).

\section{Acknowledgements}

This work was supported by grants from Fundación Nuestros Hijos (C.S.) and FONDECYT 1160426 (M.J.F.). We are also thankful to Dr. Alejandro Molinelli for helpful discussion and editorial revisions to the manuscript.

\section{Author Contributions}

N.E. participated in the samples processing, genotype determination, data analysis and writing of the manuscript. J.G. carried out voriconazole plasma concentration by HPLC analytical method for determination of voriconazole. D.N. participated in the design of the study. C.S. participated in the design of the study, data analysis, manuscript writing and final approval of the manuscript. M.J.F. participated in the design of the study, data analysis, manuscript writing and final approval of the manuscript. All authors read and approved the final manuscript.

\section{Additional Information}

Supplementary information accompanies this paper at https://doi.org/10.1038/s41598-019-45345-2.

Competing Interests: The authors declare no competing interests.

Publisher's note: Springer Nature remains neutral with regard to jurisdictional claims in published maps and institutional affiliations.

(c) (i) Open Access This article is licensed under a Creative Commons Attribution 4.0 International

License, which permits use, sharing, adaptation, distribution and reproduction in any medium or format, as long as you give appropriate credit to the original author(s) and the source, provide a link to the Creative Commons license, and indicate if changes were made. The images or other third party material in this article are included in the article's Creative Commons license, unless indicated otherwise in a credit line to the material. If material is not included in the article's Creative Commons license and your intended use is not permitted by statutory regulation or exceeds the permitted use, you will need to obtain permission directly from the copyright holder. To view a copy of this license, visit http://creativecommons.org/licenses/by/4.0/.

(c) The Author(s) 2019 